\title{
Un diagnostic de maladie coe- liaque peut en cacher un autre
}

\author{
A Diagnosis of Coeliac Disease can Hide an Alternative Diagnosis
}

\section{Présentation du cas}

Une patiente de 68 ans chute à domicile en novembre 2008 sans pouvoir se relever dans un contexte de dénutrition et d'asthénie généralisée apparues en quelques mois. Elle mentionne un état fébrile à $38^{\circ}$ accompagnant une toux grasse et la récidive de douleurs abdominales de type ballonnement, associées à des diarrhées et des nausées sans vomissement. Ses antécédents révèlent une maladie coeliaque diagnostiquée en 2005 , suite à une perte pondérale de $17 \mathrm{~kg}$ en 4 mois (jamais repris dès lors), uniquement sur la base d'une réponse anamnestique au régime sans gluten, malgré une oesogastro-duodénoscopie (OGD) normale et l'absence d'anticorps anti-transglutaminase. En décembre 2007, elle est réhospitalisée en raison d'une récidive de symptômes digestifs malgré un régime bien conduit qui est attribuée à une pullulation bactérienne. L'OGD à ce moment montre l'apparition d'une atrophie villositaire du duodenum qui va s'aggraver lors d'une troisième OGD effectuée en mars 2008. Un asthme bronchique avec infections multiples dans l'enfance se complique de bronchiectasies diagnostiquées sur un CT effectué en 2007. Une leucémie lymphoïde chronique
Abbreviations:
OGD oeso-gastro-duodénoscopie
LLC leucémie lymphoïde chronique
Ig immunoglobuline
CVID immunodéficience commune variable («common variable immunodeficiency")

(LLC) diagnostiquée en 1992, stable durant 10 ans, est traitée en 2003-2004 par 4 cycles de chimiothérapie de cyclophosphamide, rituximab et fludarabine avec une rémission complète jusqu'à ce jour. Durant les mois hivernaux, en raison d'un déficit en immunoglobulines (Ig) (toutes les classes) attribué à la LLC, son oncologue traitant a débuté une substitution en immunoglobuline dès 2000. La patiente est également connue pour une lymphopénie T CD4 + sévère (19 cells $/ \mathrm{mm}^{3}$; norme $490-1640$ ) dès 2004 compliquée de plusieurs infections virales (zona étendu, dermatomes de D12 à L1 en 2006, Herpès type I étendu au niveau endo-buccal en 2008). Le suivi lymphocytaire effectué en ambulatoire depuis 1992 en raison de la LLC évoque une lymphopénie secondaire à la chimiothérapie.

L'examen d'entrée met en évidence une cachexie (BMI à $17 \mathrm{~kg} / \mathrm{m}^{2}$ ) avec une amyotrophie. L'abdomen est distendu, diffusément douloureux et les bruits sont métalliques. L'auscultation pulmonaire révèle des râles bronchiques grossiers sur toutes les plages et quelques sibilances expiratoires. La patiente est afébrile.

Le laboratoire d'entrée est résumé dans le tableau 1.

En résumé, nous sommes en présence d'une patiente sévèrement dénutrie qui, sur le plan clinique, présente des signes d'iléus et une possible surinfection pulmonaire. Sur le plan biologique, on constate un important syndrome inflammatoire, des signes de malabsorp- tion biologique et une immunosuppression tant cellulaire qu'humorale.

\section{Examens complémentaires et diagnostic différentiel}

Sur le plan infectieux, les cultures de selles, la recherche de parasite et les hémocultures sont stériles. Les cultures d'expectorations sont positives pour un Pseudomonas Aeruginosa multisensible. Sur le plan immunologique, une répartition lymphocytaire confirme des lymphocytes T CD4+ effondrés à 157 cells $/ \mathrm{mm}^{3}$ (norme 490-1640), les autres types de lymphocytes sont dans la norme. Le test HIV est négatif. Une prophylaxie contre le Pneumocystis Jiroveci par Bactrim ${ }^{\circledR}$ est débutée.

Sur le plan digestif, un CT-scan abdominal confirme un ileus d'origine paralytique suite à un abus de lopéramide en raison des diarrhées chroniques. Alors que la patiente certifie suivre un régime sans gluten et sans lactose strict, l'OGD montre une aggravation de l'atrophie villositaire. L'examen histologique met en évidence une hyperplasie des cryptes et lésions lympho-épithéliales multiples. On constate une augmentation des lymphocytes intra-épithéliaux et une raréfaction voire absence de plasmocytes (Fig. 1 et 2).

Les multiples biopsies digestives, l'entéro-IRM et les examens bactériologiques et parasitaires ne fournissent pas d'argument en faveur d'un lymphome digestif, d'une infiltration digestive compatible 
avec une récidive de la LLC, d'un adénocarcinome grêle, d'une gastro-entérite à éosinophiles, d'une sprue collagène, d'un Whipple, d'une pullulation bactérienne, d'une tuberculose digestive ou d'une infection par Giardia Lamblia.

\section{Diagnostic}

La clé du diagnostic nous est donnée par l'examen histologique de la muqueuse duodénale associé à l'anamnèse d'infections pulmonaires multiples dans l'enfance, aux bronchiectasies mises en évidence sur le CT thoracique et au déficit en Ig (Tab. 2). Ce tableau clinique et biologique signe une immunodéficience commune variable (CVID). La raréfaction des plasmocytes intra muqueux à l'histologie permet de différencier la CVID de la maladie coeliaque réfractaire [1,2] (Tab. 3).

\section{Evolution}

Suite au diagnostic et du fait de l'atteinte pulmonaire, la substitution en Ig a été renforcée avec suivi des taux. Un traitement par corticoïdes ou immunosuppresseur pour l'atteinte digestive est contre-indiqué au vu de la lymphopénie T CD4+ concomitante. Un traitement antibiotique pourrait être envisagé lors d'une récidive des symptômes digestifs dans l'hypothèse d'une possible pullulation bactérienne récidivante. Le régime sans gluten a été stoppé sans récidive des symptômes.

\section{Discussion}

Ce diagnostic tardif est dû probablement aux multiples affections concomitantes pouvant mimer certains symptômes et éléments biologiques de la CVID. Les multiples infections pulmonaires par le passé et le développement de bronchiectasies avaient été attribués à un asthme bronchique. Les symptômes digestifs avaient été attribués à une maladie coeliaque «diagnostiquée» uniquement sur la base d'une réponse anamnestique transitoire au régime sans gluten. La LLC
Tab. 1: Valeurs de laboratoire à l'entrée (en gras, en dehors de la norme).

\begin{tabular}{|c|c|c|}
\hline Hémogramme et crase & Patiente & Normes \\
\hline Hémoglobine $(\mathrm{g} / \mathrm{l})$ & 83 & 117-157 \\
\hline MCV (fl) & 72 & 81-99 \\
\hline $\mathrm{MCH}(p g)$ & 21.3 & $27-34$ \\
\hline $\mathrm{MCHC}(\mathrm{g} / \mathrm{l})$ & 297 & $310-360$ \\
\hline Leucocytes (G/I) & 3.2 & $4.0-10.0$ \\
\hline Lymphocytes absolus (G/l) & 0.5 & $1.5-4.0$ \\
\hline TP (\%) [INR] & $50[1.4]$ & $80-120$ \\
\hline aPTT (sec) & 36 & $20-30$ \\
\hline \multicolumn{3}{|l|}{ Chimie clinique } \\
\hline Sodium (mmol/l) & 138 & $135-145$ \\
\hline Potassium (mmol/l) & 2.5 & $3.5-4.6$ \\
\hline Phosphate (mmol/l) & 0.63 & $0.8-1.4$ \\
\hline Calcium corrigé (mmol/l) & 2.33 & $2.1-2.5$ \\
\hline Magnésium total $(\mathrm{mmol} / \mathrm{l})$ & 0.57 & $0.6-1.0$ \\
\hline Protéine totale (g/l) & 57 & $59-80$ \\
\hline Albumine (g/l) & 27 & $35-52$ \\
\hline CRP (mg/l) & 305 & $<10$ \\
\hline Ferritine $(\mu \mathrm{g} / \mathrm{l})$ & 92 & $30-300$ \\
\hline Fer sérique ( $\mu \mathrm{mol} / \mathrm{l})$ & 3.0 & $10.7-21.4$ \\
\hline Zinc ( $\mu \mathrm{mol} / \mathrm{l})$ & 10.0 & $10.7-17.5$ \\
\hline Folate (nmol/l) & 3.1 & $>5.3$ \\
\hline $25-\mathrm{OH}-\mathrm{Vit} \mathrm{D}_{3}(\mu \mathrm{g} / \mathrm{l})$ & 9.7 & $8.8-44.2$ \\
\hline
\end{tabular}

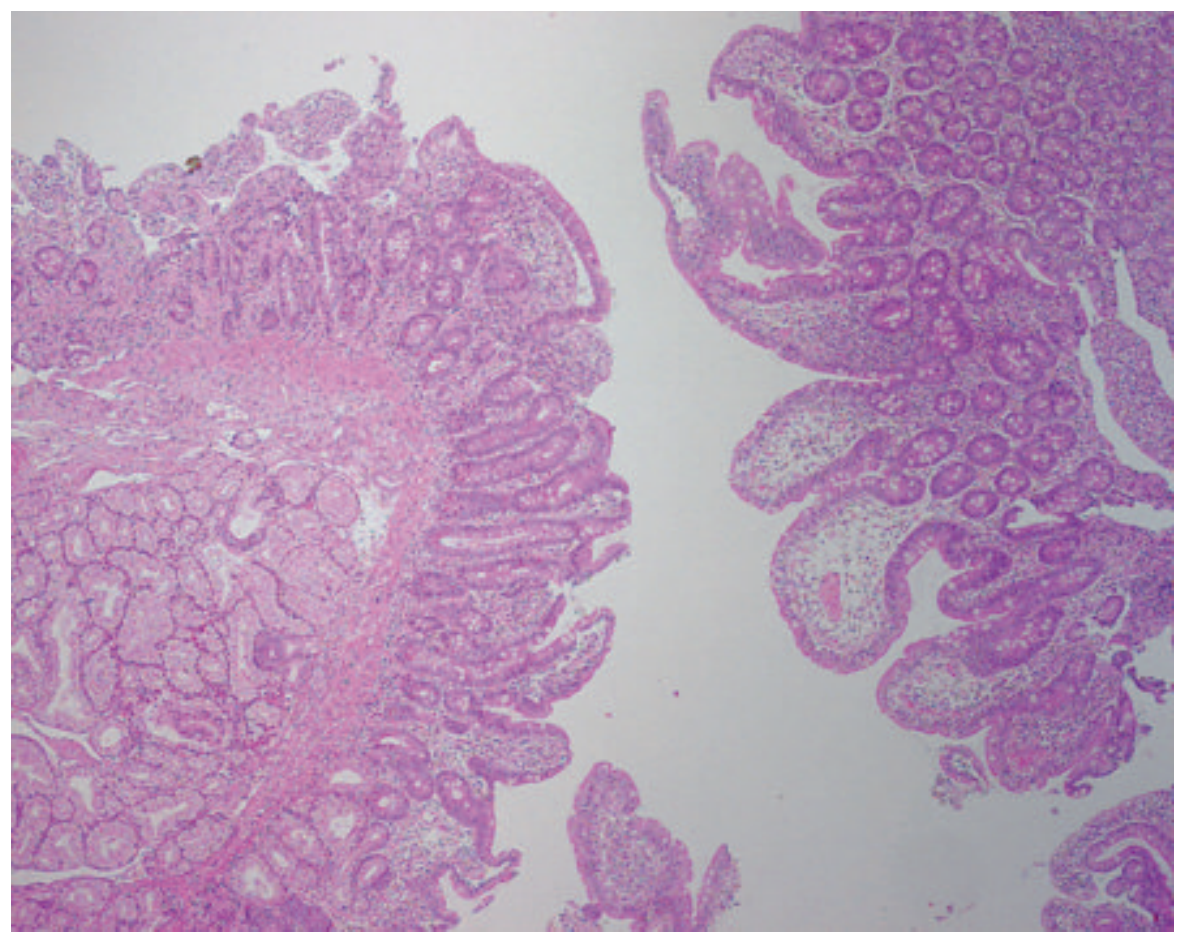

Fig. 1: Examen histologique de la muqueuse duodénale avec atrophie et œdème villositaire, $40 \times$. 
Tab. 2: Taux d'immunoglobulines sériques (en gras, en dehors de la norme).

$\begin{array}{lcc}\text { Immunoglobulines } & \text { Patiente } & \text { Normes } \\ \text { IgG (g/l) } & 0.96 & 7.0-14.5 \\ \text { - sous-classe IgG1 } & \mathbf{0 . 5 8} & 5.2-12.7 \\ \text { - sous-classe IgG2 } & \mathbf{0 . 2 0} & 1.43-5.6 \\ \text { - sous-classe IgG3 } & \mathbf{0 . 0 3} & 0.28-1.05 \\ \text { - sous-classe IgG4 } & 0.015 & 0.011-1.04 \\ \text { IgA } & <0.06 & 0.71-4.07 \\ \text { IgM } & <0.04 & 0.34-2.41 \\ \text { IgE (Ui/ml) } & 3 & 5-50\end{array}$

Tab. 3: Diagnostic différentiel des atrophies villositaires (AV) gastro-intestinales (adapté selon [10,11]).

$\begin{array}{ll}\text { Maladie coeliaque } & \\ \text { Causes infectieuses et } & \text { - Sprue tropicale } \\ \text { inflammatoires } & \text { - Maladie de Whipple } \\ \text { - Maladie de Crohn } & \text { - Giardiase } \\ \text { - Cryptosporidiose intestinale } \\ \text { - Coccidiose: Isospora belli et hominu } \\ \text { - Schistosomiase } \\ \text { - Strongylö̈dose } \\ \text { - Microsporidiose } \\ \text { - Pullulation microbienne, tel le syndrome de l'anse stagnante } \\ \text { - Entéropathie auto-immune } \\ \text { - Réaction du greffon contre l'hôte et rejet de greffe intestinale } \\ \text { - Déficit sélectif en IgA } \\ \text { - Immunodéficience commune variable } \\ \text { - Atrophie villositaire iléale primitive }\end{array}$

Maladie des chaînes alpha

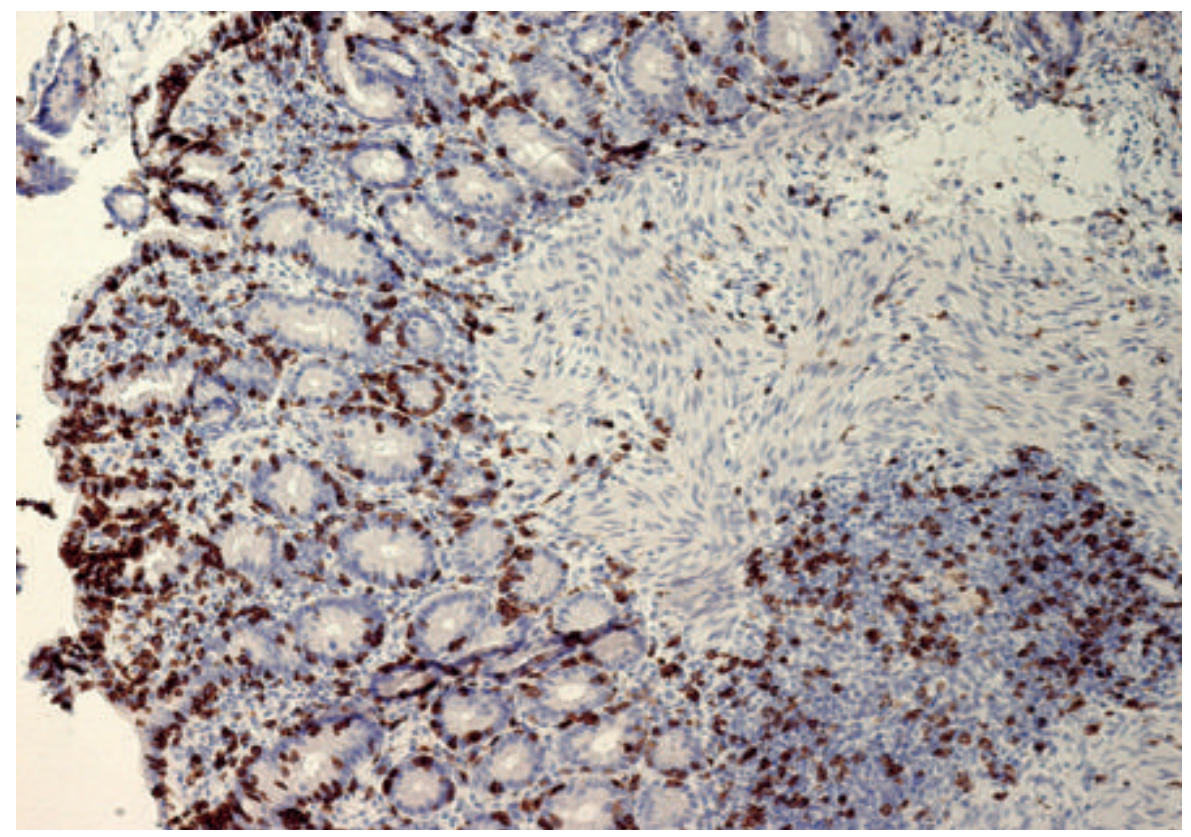

Fig. 2: Examen histologique de la muqueuse duodénale: Immunomarquage CD 8+ (lymphocytes T cytotoxiques), 100 $\times$, nombreux lymphocytes T, CD8+ intraépitheliaux et dans un follicule lymphocytaire (à droite, partie centrale). est bien connue pour entraîner une carence en Ig, pouvant se prolonger même après rémission bien que l'évolution devrait montrer une récupération progressive. Alternativement, nous ne pouvons exclure la possibilité que la LLC dont a souffert la patiente soit une complication possible d'une CVID non diagnostiquée d'apparition précoce. En effet, les prolifération polyclonales/ clonales lymphocytaires sont des complications connues et bien décrites d'une CVID [3]. Il est possible que la lymphopénie $\mathrm{T}$ CD4+ sévère induite par la chimiothérapie ait participé à l'augmentation du nombre des infections pulmonaires mais également virales observées dans les dernières années. Quand à l'origine de cette lymphopénie, une explication alternative pourrait être l'appartenance à un sous-groupe distinct de CVID définit par son association à un déficit sévère en lymphocytes T CD4+, la «Late-Onset Combined Immune Deficiency (LOCID)», décrit récemment dans les efforts de classification de cette maladie si hétérogène [4]. Cependant, le suivi régulier des lymphocytes et la concordance dans le temps évoquent fortement une conséquence directe du traitement chimiothérapeutique dans notre cas. Une immunophénotypisation des lymphocytes T permettrait éventuellement de différencier les sous-groupes, mais n'a pas été réalisée. Finalement, il est possible que le rituximab ait encore aggravé l'hypogammaglobulinémie. Ce dernier traitement de même que la LLC entrent dans le diagnostic différentiel de la symptomatologie présentée par notre patiente, bien que la littérature n'ait jamais décrit d'immunodéficience humorale acquise ayant entraîné les manifestations typiques de CVID digestives et pulmonaires ce pour quoi, nous ne les retenons pas.

\section{Définition de l'immunodéficience commune variable}

La CVID est caractérisée par une diminution de la concentration sérique des 
IgG associée à une baisse des IgA et/ou des IgM avec une réponse faible ou absente à l'immunisation et des manifestations cliniques hétérogènes ( $\mathrm{cf}$ cidessous) en l'absence de tout autre état d'immunodéficience avéré. La CVID correspond en partie à un diagnostic d'exclusion [5]. Du point de vue de la pathogenèse, il s'agit d'une différenciation altérée des lymphocytes B et d'un déficit dans la synthèse des Ig.

\section{Epidémiologie}

L'incidence de la CVID s'élève à 1/25000 avec une prévalence augmentée chez les nordiques. La maladie peut se déclarer à tout âge avec toutefois deux pics, le premier entre 1 et 5 ans, le deuxième entre 18 et 25 ans. Le début des symptômes se situe à 23 ans pour les hommes et 28 ans pour les femmes en moyenne [5].

\section{Messages-clés}

- La CVID est le déficit immunitaire primaire le plus fréquent après le déficit sélectif en IgA.

- Le diagnostic est souvent posé tardivement et des atteintes irréversibles peuvent déjà s'être développées.

- Il faudrait suspecter ce diagnostic chez chaque patient présentant des infections récurrentes ou autre manifestation clinique typique déjà au cabinet du généraliste.

- Le diagnostic de CVID doit aussi être évoqué chez les adultes et même des patients plus âgés.

\section{Key messages}

- Die CVID ist nach dem selektiven IgA-Mangel der zweithäufigste primäre Immundefekt.

- Eine zu späte Diagnose kann irreversible Organschäden zur Folge haben.

- Alle Patienten mit rezidivierenden Infektionen und/oder anderen typischen klinischen Manifestationen sollten vom Hausarzt auf eine allfällige CVID abgeklärt worden.

- Die CVID kann auch erst im Erwachsenen- oder sogar fortgeschrittenen Alter auftreten.

Tab. 4: Manifestations cliniques de l'immunodéficience commune variable.

Infections aiguës, chroniques ou récurrentes

\section{Maladies pulmonaires chroniques}

(30\% au diagnostic et jusqu'à 50\% avec l'évolution) à hautes morbi-/mortalité.

Un déficit des lymphocytes B mémoire, un taux sérique bas de "mannose-binding lectin» (> réponse immunitaire innée) et une infection par HHV8 pourrait expliquer ces pathologies. Lymphoprolifération

\section{Hépatopathie (10\%)}

Maladies autoimmunes (22\%)

\section{Néoplasies}

\section{Granulomes non-caséeux (8-20\%)}

Atteintes digestives (+/-50\%)
Bactériennes: germes pyogènes principalement (sino-pulmonaires: Pneumcoque, Haemophilus et Mycoplasme), infections digestives (Yersinia et Campylobacter)

Virales (y.c. si fonction lymphocytaire T préservée): Entérovirus (infections chroniques), Herpès simplex et Herpès Zoster

Protozoaires: infections digestives (Giardia Lamblia)

Bronchiectasies: Complication des infections pulmonaires récurrentes

Maladies granulomateuses

Bronchiolite folliculaires, hyperplasies lymphoïdes, lymphomes pulmonaires

Pneumonies interstitielles

Polyadénopathies, splénomégalie, hyperplasie lymphonodulaire intestinale (= hyperplasie folliculaire réactionnelle)

Phosphatase alcaline souvent élevée, origine inconnue (suspicion d'une hyperplasie nodulaire régénérative), association fréquente avec maladies auto-immunes Polyarthrite rhumatoïde

Hémopathies (anémie hémolytique autoimmune, anémie pernicieuse de Biermer) Neuropathies (Guillain-Barré)

Endocrinopathies (thyroïde)

Carcinome pulmonaire

Lymphome non-hodgkiniens (incidence augmentée 300×)

Peau, tractus digestif, autres organes (tous les organes lymphoïdes et solides)

Atrophie villositaire "sprue-like illness" (malabsorption), hyperplasie lymphoïde nodulaire, infections (giardiase chronique), entéropathie exsudative, intolérance au lactose, gastrite atrophique/anémie pernicieuse, maladies inflammatoires (colite ulcérative, proctite ulcérative, pseudo-maldie de Crohn) 


\section{Clinique}

L'hétérogénéité des manifestations cliniques de la maladie explique le terme «variable» de CVID et entraîne souvent un diagnostic tardif, à savoir une moyenne de 5 à 7 ans après le début des symptômes [6]. L'examen clinique met en général en évidence des signes de maladie chronique (perte de poids chez l'adulte, retard de croissance chez l'enfant) associés à des manifestations cliniques variables qui sont indiquées avec leurs fréquences dans le tableau 4.

Nous ne détaillerons ici que l'atteinte digestive (environ $50 \%$ des patients). Elle se présente sous différentes formes: par ordre de prévalence, les maladies inflammatoires gastro-intestinales (colite ulcérative, pseudo maladie de Crohn, proctite ulcérative), l'atrophie villositaire «sprue-like illness», l'hyperplasie lymphoïde nodulaire, l'anémie pernicieuse, la giardiase chronique, l'entéropathie exsudative ou la malabsorption non spécifique. Dans le contexte de diarrhées avec malabsorption et perte de poids, une carence en vitamines et électrolytes est souvent retrouvée [7]. La pathogenèse encore peu connue semble aussi liée à une baisse des lymphocytes $\mathrm{B}$ mémoire. La biopsie intestinale révèle fréquemment un déficit en plasmocytes dans le tractus gastro-intestinal et des agrégats lymphoïdes [1].

Le laboratoire, en l'absence de pathologies spécifiques associées, peut être sans particularité, hormis une diminution des Ig et des protéines totales. Les taux de lymphocytes $B$ et $T$ sont généralement normaux, mais une diminution des lymphocytes $B$ mémoire circulants (CD27+ lymphocytes B), un déficit de la fonction des lymphocytes $\mathrm{T}$ de même que des taux de cytokines abaissés peuvent parfois être retrouvés. Dans l'évolution de la maladie, une lymphopénie légère, en particulier $\mathrm{T} \mathrm{CD} 4+$ peut apparaitre $[2,6]$.
Tab. 5: Diagnostic différentiel des hypogammaglobulinémies.

\begin{tabular}{|c|c|}
\hline \multicolumn{2}{|l|}{ Hypogammaglobulinémies primaires } \\
\hline Immunodéficience commune variable & $\begin{array}{l}\text { La plus fréquente: déficit IgG, } A \text { et/ou } M \text {, réponse } \\
\text { déficitaire à l'immunisation active }\end{array}$ \\
\hline $\begin{array}{l}\text { Hypogammaglobulinémie transitoire } \\
\text { de l'enfant }\end{array}$ & $\begin{array}{l}\text { Normalisation des Ig à } 24 \text { mois, bon pronostique, } \\
\text { immunité cellulaire normale et bonne réponse aux } \\
\text { vaccins }\end{array}$ \\
\hline Agammaglobulinémie & $\begin{array}{l}\text { Liée à l'X ou autosomale récessive, début dans } \\
\text { petite enfance, hypoplasie du tissu lymphoïde, } \\
\text { lymphocytes B diminués ou absents }\end{array}$ \\
\hline Hyper-IgM-syndrome & $\begin{array}{l}\text { Différentes anomalies génétiques connues, } 2 / 3 \text { lié à } \\
\text { I'X, IgM normales voire augmentées, IgG, A et E } \\
\text { diminuées, infections opportunistes (Pneumocystis } \\
\text { Jiroveci) liées au déficit des lymphocytes T associé }\end{array}$ \\
\hline Maladie lymphoproliférative liée à l’X & $\begin{array}{l}\text { Lymphoprolifération par dysrégulation de l'immu- } \\
\text { nité, insuffisance hépatique, hypogammaglobuliné- } \\
\text { mie et/ou lymphome, événement déclenchant = } \\
\text { infection par virus EBV }\end{array}$ \\
\hline Immunodéficience combinée & Lymphopénie T avec taux de lymphocytes B variable \\
\hline Déficit en anticorps sélectifs & $\begin{array}{l}\text { Taux d'Ig normal, infections sino-pulmonaires } \\
\text { récurrentes, incapacité à produire de Ac sélectifs en } \\
\text { réponse à un vaccin }\end{array}$ \\
\hline Déficit en sous-classe d'IgG & $\begin{array}{l}\text { Déficit dans une ou plusieurs des sous-classes d'Ig, } \\
\text { infections sino-pulmonaires à répétition, taux d'Ig A, } \\
\text { M et G totaux normaux }\end{array}$ \\
\hline \multicolumn{2}{|l|}{ Hypogammaglobulinémies secondaires } \\
\hline Défaut de production & $\begin{array}{l}\text { - néoplasie: thymome, leucémie lymphoïde chroni- } \\
\text { que, lymphome } \\
\text { - médicamenteux: immunosuppresseurs, rituximab } \\
\text { et anti-épileptiques (carbamazepine, phenytoin, } \\
\text { acide valproïque, chlorpromazine, lamotrigine,...) } \\
\text { - infections virales (EBV, CMV, rubéole congénitale } \\
\text { et parvovirus B19) } \\
\text { - maladies systémiques avec dépression médullaire }\end{array}$ \\
\hline Augmentation de la perte & $\begin{array}{l}\text { - entéropathies exsudatives } \\
\text { - syndrome néphrotique } \\
\text { - grands brûlés } \\
\text { - autres traumatismes avec perte de fluides } \\
\text { importante }\end{array}$ \\
\hline
\end{tabular}

\section{Diagnostic}

Une CVID devrait être recherchée chez tout patient présentant des infections à répétition ou une voire plusieurs des manifestations non infectieuses associées (Tab. 4) .Le diagnostic est posé par un taux sérique des Ig diminué de deux déviations standards au-dessous de la moyenne, ajusté à l'âge, lors de deux dosages distincts des IgG, A et/ou M. La réponse aux vaccinations actives peut être évaluée, sauf en cas d'Ig $<200 \mathrm{mg} / \mathrm{dl}$ où l'on extrapole qu'elle sera déficiente.
Dans la plupart des cas, la réponse aux vaccins protéiques et polysaccharidiques est déficitaire.

\section{Diagnostic différentiel}

Le diagnostic différentiel des hypogammaglobulinémies se pose entre les causes primaires et secondaires. L'anamnèse (y compris familiale) et le laboratoire permettent en général de poser le diagnostic (Tab. 5). 


\section{Traitement}

Tout patient atteint de CVID doit recevoir une substitution en Ig, afin de réduire le nombre d'infections, les hospitalisations et l'utilisation d'antibiotiques, sans que cela élimine complètement le risque infectieux particulièrement au niveau digestif et sino-pulmonaire. Cette substitution semble aussi permettre de ralentir l'évolution de l'atteinte pulmonaire (bronchectasie) et avoir un effet protecteur contre le développement des maladies auto-immunes. Cet effet protecteur n'a pas été retrouvé pour les néoplasies et pour les maladies granulomateuses (Tab. 4).

En pratique, les Ig, d'une demi vie d'environ 3 semaines, se substituent iv ou sc. Le «steady state» est atteint après 3 à 6 mois de substitution et se mesure par un taux d'Ig 6 mois après la première dose puis chaque 6 mois. Un taux normal est visé excepté lors de pathologies pulmonaires associées ou lors d'atteintes hématologiques auto-immunes sous substitution, qui requièrent un taux plus élevé. La dose de départ est de 300$400 \mathrm{mg} / \mathrm{kg}$ par mois à augmenter selon la réponse $[2,5,8,9]$.

Sauf en cas de lymphocytes T CD4+ $<200$ cells/ml (prophylaxie pour le Pneumocystis Jiroveci), d'une médication immunosuppressive ou de corticoïdes systémiques, l'antibiothérapie prophylactique n'est pas recommandée. Une antibiothérapie thérapeutique précoce et prolongée par rapport aux durées habituelles est par contre essentielle pour prévenir le développement des bronchiectasies.

Le développement des complications associées doit être surveillé attentivement et un traitement prescrit si possible. Dans l'atteinte gastro-intestinale non infectieuse, les carences électrolytiques, protéiniques, vitaminiques et en oligo-élément doivent être substituées et lors d'entéropathies sévères, un traitement anti-inflammatoire (ex. mesalazine) ou immunosuppresseur de courte durée (glucocorticoïdes, azathioprine, 6-mercaptopurine) doit être prescrit. Les maladies auto-immunes semblent prévenues par la substitution en Ig. Les infiltrations granulomateuses nécessitent un traitement anti-inflammatoire ou immunosuppresseur. Un suivi attentif permettra de détecter les néoplasies les plus fréquemment associées de manière précoce (Tab. 4 ).

\section{Pronostic}

Depuis l'instauration de la substitution en Ig, la mortalité liée aux infections bactériennes aiguës a beaucoup diminué. Actuellement, elle semble plutôt liée aux complications pulmonaires chroniques et aux lymphomes associés $[8,9]$. Des facteurs de mauvais pronostic sont un taux très bas d'IgG, une réponse faible des lymphocytes $\mathrm{T}$ à la phytohémagglutinine, un pourcentage bas de lymphocytes $B$ périphériques de même que la présence de bronchiectasies, d'entéropathie, d'une infiltration polyclonale lymphocytaire (granulomes, hépato-/splénomégalie, adénopathies; liée à une augmentation des lymphomes) et d'auto-immunité. Le pourcentage de cellules B mémoire circulant est prédicateur du risque d'apparition de certaines complications.

\section{Remerciements}

Les auteurs tiennent à remercier le Professeur Urs Scherrer du Département de Médecine interne, CHUV pour sa lecture critique du manuscrit et ses précieux conseils.

\section{Résumé}

La CVID est le déficit immunitaire primaire le plus fréquent après le déficit sélectif en IgA. Par la variabilité de ses manifestations cliniques et son âge de déclaration fluctuant, elle mime d'autres pathologies et se retrouve souvent diagnostiquée tardivement à un moment où les patients souffrent de complications irréversibles qui auraient pu être prévenues par un diagnostic et traitement précoce. Par conséquence, le médecin généraliste doit évoquer ce diagnostic chez tout patient présentant des infections à répétitions ou autres manifestations cliniques typiques de la CVID.

Mots-clés: immunodéficience commune variable - atrophie villositaire immunoglobulines - infections récurrentes

\section{Abstract}

The Common variable Immunodeficiency (CVID) is next to the selective IgA-deficiency the most frequent primary immunodeficiency syndrome. Because of its variable clinical manifestations and age of declaration, CVID can mimic different other pathologies and is therefore frequently diagnosed in a later stage of the disease. However, as a consequence of late diagnosis, irreversible organ damage can have occurred which could have been prevented by early treatment. Therefore, early diagnosis of CVID by the general practitioner in patients with recurrent infections or other typical clinical manifestations is of great importance.

Key words: common variable immunodeficiency - villous atrophy - immunoglobulines - recurrent infections

\section{Zusammenfassung}

Die Common Variable Immunodeficiency (CVID) ist nach dem selektiven IgA-Mangel der häufigste primäre Immundefekt. Aufgrund der unterschiedlichen Symptomatik und des variablen Manifestationsalters kann die CVID verschiedene andere Pathologien imitieren und wird deshalb häufig erst im späteren Krankheitsverlauf diagnostiziert. Eine zu späte Diagnose kann irreversible Organ- 
schäden zur Folge haben, wovon die meisten durch eine frühe Behandlung vermieden werden können. Deshalb ist eine frühe Diagnose durch den Hausarzt bei Patienten mit rezidivierenden Infekten oder anderen typischen klinischen Manifestationen von grosser Wichtigkeit.

Schlüsselwörter: Common variable immunodeficiency - Zottenatrophie Immunoglobuline - Infektionen, rezidivierende

\section{Adresse de correspondance}

Marie Yersin

Médecin-assistante

Département de médecine interne

CHUV

1011 Lausanne

marie.yersin@chuv.ch

\section{Bibliographie}

1. Daniels JA, Lederman HM, Maitra A, et al.: Gastrointestinal tract pathology in patients with common variable immunodeficiency (CVID): a clinicopathologic study and review. Am J Surg Pathol 31:1800-1812, 2007

2. Sneller MC, Strober W, Eisenstein E, et al.: NIH conference. New insights into common variable immunodeficiency. Ann Intern Med 118:720-730, 1993

3. Mellemkjaer L, Hammarstrom L, Andersen V, et al.: Cancer risk among patients with IgA deficiency or common variable immunodeficiency and their relatives: a combined Danish and Swedish study. Clin Exp Immunol 130:495-500, 2002

4. Malphettes $M$, Gerard L, Carmagnat $M$, et al.: Late-onset combined immune deficiency: a subset of common variable immunodeficiency with severe T cell defect. Clin Infect Dis 49:13291338,2009

5. Webster ADB: Common variable Immunodeficiency. Immunology and Allergy Clinics of North America 21:1-22, 2001

6. Cunningham-Rundles C, Bodian C: Common variable immunodeficiency: clinical and immunological features of 248 patients. Clin Immunol 92:34-48, 1999

7. Khodadad A, Aghamohammadi A, Parvaneh N, et al.: Gastrointestinal manifestations in patients with common variable immunodeficiency. Dig Dis Sci 52:2977-2983, 2007

8. Aghamohammadi A, Pouladi N, Parvaneh N, et al.: Mortality and morbidity in common variable immunodeficiency. J Trop Pediatr 53:32-38, 2007

9. Quinti I, Soresina A, Spadaro G, et al.: Long-term follow-up and outcome of a large cohort of patients with common variable immunodeficiency. J Clin Immunol 27:308-316, 2007

10. Patey-Mariaud De Serre N, Verkarre V, Cellier C, et al.: [Etiological diagnosis of villous atrophy]. Gastroenterol Clin Biol 24:436-446, 2000

11. Shah VH, Rotterdam H, Kotler DP, et al.: All that scallops is not celiac disease. Gastrointest Endosc 51:717-720, 2000 